\title{
Dengue epidemic in Malaysia: Not a predominantly urban disease anymore
}

\author{
Nor Azila Muhammad Azami ${ }^{1}$, Sharifah Azura Salleh², Hui-min Neoh', Syed Zulkifli Syed Zakaria ${ }^{1,3}$ and \\ Rahman Jamal ${ }^{1,3^{*}}$
}

\begin{abstract}
Background: Dengue infection has been an important and serious public health concern in Malaysia ever since its first reported case here in 1902. Nevertheless, to our knowledge, no nationwide investigation has been carried out to determine the actual magnitude of dengue endemicity in the Malaysian population. In this study, we describe a cross sectional seroepidemiology study of dengue lgG seroprevalence in the Malaysian adult population.
\end{abstract}

Findings: From 1000 subjects (35-74 years old), 91.6\% subjects were found to be dengue seropositive. Age is found to be a significant risk factor associated with dengue seroposivity, where the seroprevalence increased with every 10 year increase in age. Nevertheless, gender and ethnicity did not have an effect. Interestingly, there were similar seroprevalence rates between urban and rural samples, showing that dengue is presently not confined to urban areas in Malaysia.

Conclusions: High dengue lgG seropositivity found in the population is an indication that dengue might be endemic in Malaysia for a long time into the future. Public awareness, proper vector control and vigilant surveillance are critical to keep the infection rates low and to prevent outbreaks.

\section{Findings}

\section{Background}

Dengue infection has been an important public health concern in Malaysia ever since its first reported case here in 1902 [1]. The virus (DENV) is transmitted by the mosquito Aedes aegypti, which is usually found in urban areas [2]. Across the world, about 100 million cases of dengue infection and 500000 cases of dengue haemorrhagic fever (DHF) with 12000 deaths were reported per year [3].

Clinical manifestations of dengue infection ranges from non-specific febrile illness to classical dengue fever (DF), DHF and dengue shock syndrome (DSS) [4]. Reported cases that are accounted for by public health statistics are usually secondary infections, where symptoms of DF, DHF or DSS might be present. These clinical manifestations are all thought to be a consequence of a heightened immune response due to cross-reactive $\mathrm{T}$-cell responses and/or enhancing dengue antibody

\footnotetext{
* Correspondence: rahmanj@ppukm.ukm.my

'UKM Medical Molecular Biology Institute, Universiti Kebangsaan Malaysia, Malaysia

Full list of author information is available at the end of the article
}

[5-7]. On the other hand, first infection by DENV is usually subclinical and can present atypically as an undifferentiated febrile illness [8].

Malaysia, with a population of approximately 27.7 million and a population density of 84 per sq. $\mathrm{km}$, has continuously recorded rising annual cases of dengue infection since 1980. Major, national dengue outbreaks were reported in 1974, 1978, 1982 and 1990, exhibiting a 4-year cycle [9]. According to the 2008 Health Facts by Malaysian Ministry of Health, the incidence rate of dengue was 167.76 per 100000 population with a mortality rate of 0.02 [10]. In the process of becoming a developed nation, massive infrastructure development in this country has contributed towards a high incidence of dengue infection, as urbanization is a favourable factor for Ae. aegypti breeding and subsequently facilitates the spread of DENV [11-13].

As primary exposure to the virus is usually asymptomatic, the actual magnitude of dengue infection in Malaysia may be larger than expected. We hereby conducted a cross-sectional seroepidemiology study to determine the prevalence of dengue antibodies in the Malaysian adult population. This study will be an initial

\section{() Biomed Central}


step in estimating the extent of the dengue epidemic in Malaysia.

\section{Study population}

The Malaysian Cohort (TMC) is a national project managed by our research institute (UKM Medical Molecular Biology Institute, UMBI). Initiated in 2006, this nationwide cohort recruits Malaysians aged 35 and above; its participants are considered as the best representative of the Malaysian adult population as they represent subjects from various ethnic groups, geographical locations and lifestyles. Detailed information about each participant is collected along with blood, serum, plasma, lymphocytes and urine samples. All subjects were healthy during the time of sample collection and consented towards the storage and usage of their samples for medical and epidemiological research. Ethical approval for The Malaysian Cohort has been granted by the Universiti Kebangsaan Malaysia Research Ethics committee.

There were a total of 13725 participants recruited into the TMC from 1 January 2008 until 31 December 2008. We randomly selected 1000 subjects from the initial population of 13725 using Minitab 15 software (Minitab Inc., Pennsylvania, USA), and retrieved their serum samples for our study. Socio-demographic data of the subjects such as gender, age, ethnicity, locality (urban/rural) were also retrieved from the TMC database (Table 1).

\section{Dengue IgG antibody detection}

Serum samples were measured for the presence of dengue virus-specific IgG antibodies using the PanBio Dengue IgG INDIRECT enzyme-linked immunosorbent assay (ELISA) (Inverness Medical Innovations Australia
Pty Ltd, Queensland, Australia). This kit identifies antibodies to all four DENV serotypes but cannot differentiate between each serotype. Positive result of dengue IgG antibody indicates previous exposure to DENV. Interpretation of ELISA results were made according to instructions from the manufacturer.

\section{Data Analysis}

Data analysis was performed using Statistical Package for Social Science (SPSS) 16.0 (SPSS Inc., Chicago, USA). Chi square analysis was used to determine the relationship between genders, age groups, ethnicity and locality with dengue IgG seroprevalence. Logistic regression analysis was used to determine risk factors associated with positive dengue IgG. Odd ratios and their 95\% confidence intervals were provided as estimates of the effect size and the chi-square tests were conducted at $3 \%$ level of significance while logistic regression tests were conducted at $5 \%$ level of significance.

\section{Result and Discussion}

From the 1000 subjects surveyed, 916 (91.60\%) were positive for dengue IgG, where 541 (90.17\%) females and 375 (93.75\%) males were dengue seropositive. This shows that a large proportion of the Malaysian adult population has been exposed to DENV. Ethnically, dengue IgG seroprevalence were as follows: Malay, $91.75 \%$ (523/570); Chinese, 91.37\% (339/371); Indian and others, 91.53\% (54/59) (Note: In 2008, the ethnicity ratio of recruited subjects in the TMC was comparable to the national ratio of Malay: Chinese: Indian $=6: 3: 1$ ). Chi square analysis showed no significant difference in

Table 1 Sociodemographic data of 1000 subjects whose serum samples were used in this study

\begin{tabular}{llll}
\hline Sociodemographic factor & Number of subjects* & Dengue IgG positive $^{*}$ & Dengue Seroprevalence $^{\boldsymbol{\varepsilon}}$ \\
\hline Gender & $400(40.0 \%)$ & 375 & 93.75 \\
Male & $600(60.0 \%)$ & 541 & 90.17 \\
Female & $137(13.7 \%)$ & 110 & 80.29 \\
\hline Age Range & $474(47.4 \%)$ & 439 & 92.62 \\
$35-44$ & $338(33.8 \%)$ & 319 & 94.38 \\
$45-54$ & $51(5.1 \%)$ & 48 & 94.12 \\
$55-64$ & $53(35-74)$ & & 91.75 \\
$65-74$ & $570(57.0 \%)$ & 523 & 91.37 \\
\hline Median Age, y (Range) & $371(37.1 \%)$ & 339 & 91.53 \\
\hline Ethnicity & $59(5.9 \%)$ & 54 & 90.95 \\
Malay & & 362 & 92.03 \\
Chinese & $398(39.8 \%)$ & 554 & \\
Indian \& Others & $602(60.2 \%)$ & & \\
\hline Locality & & & \\
Rural & & & \\
Urban &
\end{tabular}

*Except where indicated, values are $(n, \%)$

* Except where indicated, values are $(\mathrm{n})$

${ }^{\mathrm{f}}$ Except where indicated, values are (\%) 
dengue IgG seroprevalence between different ethnic groups $\left(\chi^{2}=0.43, \mathrm{p}=0.979\right)$ and gender $\left(\chi^{2}=4.055, \mathrm{p}\right.$ $=0.045)$; the seroprevalence rates were more than $90 \%$ for each category for both demographic factors (gender and ethnicity). This shows that a large proportion of the Malaysian adult population has been exposed to DENV; there were neither gender bias nor ethnicity preferences.

Nevertheless, we noticed that dengue IgG seroprevalence rates increased with age among our subjects, and the difference between age groups was significant $(\mathrm{p}<$ 0.001 ) (Table 1). The odds of dengue seropositivity (compared to the 35-44 years old age group) were as follows: 45-54 years old, 3.08 (95\% CI: 1.79-5.31); 55-64 years old, 3.89 (95\% CI: 2.07-7.30) ( $<<0.001)$; and 6574 years old, 3.59 (95\% CI: $1.03-12.46)(\mathrm{p}=0.045)$ (Table 2). This finding is in conjunction with reports that dengue seroprevalence will increase for every 10 year increment in age; older subjects will have a higher chance of infection $[4,14]$. As the TMC only recruits adults 35 years old and above, dengue seroprevalence in the younger population is not known; it remains to be investigated if there was a sharp rise in the seroconversion rate during childhood/young adulthood, or whether the conversion has occurred gradually.

Surprisingly, there was no difference $(\mathrm{p}=0.550)$ in the dengue IgG seroprevalence between subjects staying in urban areas $(92.03 \%, 554 / 602)$ compared to those living in rural communities (90.95\%, 362/398), even though dengue is usually described as an "urban" disease [15]. In our study, samples from "urban" areas were retrieved from the TMC control centre (based in our university hospital, UKM Medical Centre in Kuala Lumpur, Malaysia) and also town areas; while samples categorized as "rural" were collected from subjects living in villages and also settlers in palm oil plantations from the Federal Land Development Authority (FELDA) communities nationwide. Hayes et al. (1990) reported that dengue antibody seroprevalence was high in urban (66\%) compare to rural populations $(26 \%)$ or jungle areas (32-64\%) [16]. Population-based study in rural Amazonia of Brazil also showed a lower baseline DENV seropositivity rate of $18.3 \%$ [17]. Nevertheless, our study showed that there was no difference in the

\begin{tabular}{|c|c|c|c|}
\hline Covariates & Odd Ratio (OR) & 95\% Confidence Interval (Cl) & P-value ${ }^{\S}$ \\
\hline \multicolumn{4}{|l|}{ Age Range } \\
\hline $35-44$ & 1.00 & $(1.79,5.31)$ & $<0.001$ \\
\hline $45-54$ & 3.08 & $(2.07,7.30)$ & $<0.001$ \\
\hline $55-64$ & 3.89 & $(1.03,12.46)$ & 0.045 \\
\hline 65-74 & 3.59 & & \\
\hline
\end{tabular}

Bold $^{\S}$ indicates significant values, where $p<0.05$ seroprevalence between urban and rural areas. This phenomenon might be caused by rising vector populations in rural areas. A study by Chen et al. in 2005 gave an ovitrap index of $56 \%$ in a rural settlement [18]. However, in a separate study carried out 2 years later in two insular rural settlements, the ovitrap index was $63 \%$ and $80 \%$, respectively [19]. In addition to rising vector populations, improved modes of transportation which allow migration of viramic subjects from one place to another might have facilitated the spread of dengue from urban into rural areas.

The high dengue IgG seropositivity found in the Malaysian adult population (91.6\%) indicates that 9 out of 10 adults are already exposed to dengue infection. This percentage of dengue IgG prevalence is higher compared to that among adults in Singapore (45\%) and Central Brazil (29.5\%) based on house-hold surveys, but lower compared to the study among adults in Santo Domingo, Dominican Republic (98\%) [4,11,14]. The major drivers for high dengue IgG seroprevalence in Malaysia are probably demographic changes, massive urbanization in town areas, high density of vector populations such as Ae. aegypti in both urban and rural areas and changes in the usage of agricultural land in rural locations which promotes the propagation of Aedes $s p$ $[11,18]$. Lack of effective mosquito control programs may have also helped to spread the virus.

In this study, the IgG detected was considered to be specific for DENV, even though the ELISA kit used might also detect other flavivirus antibodies such as JE and Yellow Fever (YF). In Malaysia, DENV and JE viruses are two of the more common endemic flaviviruses; YF and West Nile viruses have never been isolated here. Compared to dengue, the number of JE cases in Malaysia is minimal (annual incidence of JE virus infection is 9.8 per 100000 population compared to 167.76 per 100000 population for dengue $[10,20]$. Other flaviviruses found in Malaysia such as Tembusu and Langat do not cross-react with dengue antibodies [21]. Taking it all together, our positive ELISA results were more likely to be caused by dengue IgG than antibodies from other flaviviruses.

\section{Conclusion}

To the best of our knowledge, this is the first dengue IgG seroprevalence study carried out on healthy adults in Malaysia. High dengue IgG seropositivity (91.60\%) found in the population is an indication that dengue might be endemic in Malaysia for a long time into the future. Public awareness, proper vector control and vigilant surveillance are critical to keep the infection rates down and to prevent outbreaks. It remains to be determined which dengue serotype is more dominant, and if the dengue serotypes found in this population are 
similar to or different from the current circulating serotypes in the vectors.

\section{Acknowledgements}

We wish to thank Mr. Sallehuddin Zainal, Ms. Noriza Osman, Mrs. Darna Zainuddin and Mr Anuar A Rahman of Serology Unit, Microbiology Department of UKM Medical Centre for his/her technical advice in this study. We also would like to express our gratitude to Mrs Nazihah Abdul Jalal, Ms Fatini Mohamed and staff of Biospecimen Unit of TMC Consortium for helping us in retrieving biospecimens from TMC Biobank. This study is funded by The Malaysian Cohort CAM 33-05-02-001.

\section{Author details}

${ }^{1}$ UKM Medical Molecular Biology Institute, Universiti Kebangsaan Malaysia, Malaysia. ${ }^{2}$ Department of Medical Microbiology and Immunology, Universiti Kebangsaan Malaysia. ${ }^{3}$ Secretariat, The Malaysian Cohort, Malaysia.

\section{Authors' contributions}

AA carried out the immunoassay, performed statistical analysis and drafted the manuscript. SA participated in the design of the study and data interpretation. HM participated in the design of the study and drafted the manuscript together with AA. SZ participated in the design of the study and coordination. RJ conceived the study and participated in the study design and coordination. All authors read and approved the final manuscript.

\section{Competing interests}

The authors declare that they have no competing interests.

Received: 9 December 2010 Accepted: 29 June 2011

Published: 29 June 2011

\section{References}

1. Skae FMT: Dengue fever in Penang. The British Medical Journal 1902, 2:1581-1582.

2. Smith CEG: The history of dengue in tropical Asia and its probable relationship to the mosquito Aedes Aegypti. J Trop Med Hyg 1956, 59:243-251.

3. Gubler DJ: Epidemic dengue/dengue hemorrhagic fever as a public health, social and economic problem in the 21 th century. Trends in Microbiology 2002, 10:100-103.

4. Wilder-Smith A, Foo W, Earnest A, Sremulanathan S, Paton NI: Seroepidemiology of dengue in the adult population of Singapore. Trop Med Int Health 2004, 9(2):305-8.

5. Green S, Pichyangkul S, Vaughn D, et al: Early CD69 expression on peripheral blood lymphocytes from children with dengue hemorrhagic fever. J Infect Dis 1999, 1429-1435.

6. Rothman AL, Green S, Vaughn DW, et al: Dengue hemorrhagic fever. In Factors in the emergence of arbovirus diseases. Edited by: Saluzzo JF, Dodet B. Paris, France: Elsevier; 1997:109-16.

7. Halstead SB: Observations related to pathogenesis of dengue hemorrhagic fever. VI. Hypotheses and discussion. Yale J Biol Med 1970, 42:350-362.

8. Halstead SB, Nimmannitya S, Margiotta MR: Dengue and chikungunya virus infection in man in Thailand, 1962-1964: II. Observations on disease in outpatients. Am J Trop Med Hyg 1969, 18:972-983.

9. Lam SK: Two decades of dengue in Malaysia. Trop Med 1993, 35(4):195-200,

10. Ministry of Health Malaysia. Health Facts 2008 Malaysia; 2009

11. Yamashiro T, Disla M, Petit A, Taveras D, Castro-Bello M, Lora-Orste M, Vardez S, Cesin AJ, Garcia B, Nishizono A: Seroprevalence of IgG specific for dengue virus among adults and children in Santo Domingo, Dominican Republic. The American Journal of Tropical Medicine and Hygiene 2004, 71(2):138-143.

12. Gubler DJ: Epidemic dengue/dengue hemorrhagic fever as a public health, social and economic problem in the 21 st century. Trends Microbiol 2002, 10(2):100-3.

13. Deller JJ Jr, Russell PK, Binh L: An analysis of fevers of unknown origin in American soldiers in Vietnam. Ann Intern Med 1967, 66:1129-43.
14. Siqueira JB, Martelli CM, Maciel IJ, Oliveira RM, Ribeiro MG, Amorim FP, Moreira BC, Cardoso DD, Souza WV, Andrade AL: Household survey of dengue infection in central Brazil: spatial point pattern analysis and risk factors assessment. Am J Trop Med Hyg 2004, 71(5):646-51.

15. Gubler DJ, Clark GG: Dengue/dengue hemorrhagic fever: the emergence of a global health problem. Emerging Infectious Disease 1995, 1(2):55-7.

16. Hayes CG, Phillips IA, Callahan JD, Griebenow WF, Hyams KC, Wu SJ, Watts DM: The epidemiology of dengue virus infection among urban, jungle, and rural populations in the Amazon region of Peru. Am J Trop Med Hyg 1996, 55(4):459-63.

17. Da Silva-Nunes, de Souza VA, Pannuti CS, Speranca MA, Terzian AC, Nogueira ML, Yamamura AM, Freire MS, da Silva NS, Malafronte RS, Muniz PT, Vasconcelos HB, da Silva EV, Vasconcelos PF, Ferreira MU: Risk factors for dengue virus infection in rural Amazonia: population-based cross-sectional surveys. Am J Trop Med Hyg 2008, 79(4):485-494.

18. Chen CD, Benjamin S, Saranum MM, Chiang YF, Lee HL, Nazni WA, SofianAzirun M: Dengue vector surveillance in urban residential and settlement areas in Selangor, Malaysia. Tropical Biomedicine 2005, 22(1):39-43.

19. Lim KW, Sit NW, Norzahira R, Sing KW, Wong HM, Chew HS, Firdaus R, Suria M, Mahathavan M, Nazni WA, Lee HL, McKemy A, Vasan S, S: Dengue vector surveillance in insular settlements of Pulau Ketam, Selangor, Malaysia. Tropical Biomedicine 2010, 27(2):185-192.

20. Wong SC, Ooi MH, Abdullah AR, Krishnan S, Tio PH, Pek PC, Lai BF, Mohan A, Muhi J, Kiyu A, Arif MT, Cardosa MJ: A decade of Japanese encephalitis surveillance in Sarawak, Malaysia: 1997-2006. Trop Med Int Health 2008, 13(1):52-55.

21. De Madrid AT, Porterfield JS: The flaviviruses (group B arboviruses): a cross-neutralization study. J Gen Virol 1974, 23(1):91-6.

doi:10.1186/1756-0500-4-216

Cite this article as: Muhammad Azami et al:: Dengue epidemic in Malaysia: Not a predominantly urban disease anymore. BMC Research Notes 2011 4:216.

\section{Submit your next manuscript to BioMed Central and take full advantage of:}

- Convenient online submission

- Thorough peer review

- No space constraints or color figure charges

- Immediate publication on acceptance

- Inclusion in PubMed, CAS, Scopus and Google Scholar

- Research which is freely available for redistribution

Submit your manuscript at www.biomedcentral.com/submit
C Biomed Central 